\title{
Targeted next-generation sequencing to identify genetic polymorphism associated with levetiracetam-induced psychosis
}

\author{
Jaeseong Oh', Yong-Won Shin ${ }^{2}$, Jangsup Moon ${ }^{2}$, Jihoon G. Yoon ${ }^{3}$, Sungkyoung Choi ${ }^{3}$, Yun Kim¹, \\ Kon $\mathrm{Chu}^{2}$, Sang Kun Lee ${ }^{2}$, Min Goo Lee ${ }^{3}$, In-Jin Jang ${ }^{1}$
}

${ }^{I}$ Department of Clinical Pharmacology and Therapeutics, Seoul National University College of Medicine and Hospital, Korea, ${ }^{2}$ Department of Neurology, Seoul National University College of Medicine, Korea, ${ }^{3}$ Department of

Pharmacology, Yonsei University College of Medicine, Korea

\section{Background}

Levetiracetam is a broad spectrum antiepileptic drug for the treatment of partial and generalized seizure. It is generally well tolerated in epilepsy patients, but levetiracetam-induced psychosis has been reported in some of the patients. This study aimed to investigate genetic polymorphism which is associated with levetiracetam-induced psychosis using targeted next-generation sequencing (NGS).

\section{Methods}

Twelve patients who reported levetiracetam-induced psychosis were included. Eighty patients who did not experience any psychotic adverse events during $>=2000 \mathrm{mg} /$ day administration of levetiracetam were included as the control group. Whole blood samples obtained these from ninety-two epilepsy patients were used for targeted sequencing. NGS panels were constituted with a pharmacogenomics gene panel (114 genes involved in metabolism, transport and response of various drugs) and with an epilepsy gene panel (91 genes involved in response and adverse reaction of antiepileptic drugs). Logistic regression model was used for common variant (minor allele frequency $>=5 \%$ ) analysis. Burden test and sequence kernel association tests (SKAT and SKAT-O) were performed for the rare variant (minor allele frequency $<$ 5\%) analysis.

Results

A total of 2,826 genetic variants (1,222 and 1,604 of common and rare variants, respectively) were observed from the pharmacogenomics gene panel and a total of 1,853 genetic variants (731 and 1,122 of common and rare variants, respectively) were observed from the epilepsy gene panel. None of the common variant was significantly associated with levetiracetam induced psychosis. From the rare variant analysis, three pharmacogenomic genes (SLCO2B1, UGT1A4, and PTGIS) and epilepsy genes (GABRG3, GABRA1, and CACNA1A) showed significant $\mathrm{P}$-value $(\mathrm{P}<0.05)$ in the three association tests (Burden test, SKAT, and SKAT-O). Four pharmacogenomic genes (ADRB2, ABCC4, ABCA1, and SLC47A2) and three epilepsy genes (UGT1A10, ANKK1, and GRIN2D) showed significant P-value more than one of the association tests.

Conclusions

The genetic variants identified in this study may increase susceptibility to levetiracetam-induced psychosis in epilepsy patients. Further in-vitro and in-vivo studies are required to elucidate if increasing in-vivo drug concentration and/or decreasing threshold of adverse reaction is associated with the development of levetiracetam-induced psychosis. 